\title{
Intravitreal Fasudil Monotherapy for Treatment of Refractory Diabetic Macular Edema: a Prospective, Interventional Case-series
}

Ramin Nourinia

Ophthalmic Research Center, Labbafinejad Medical Center, Shahid Beheshti University of Medical Sciences, Tehran, Iran

Seyed-Hossein Abtahi

Ophthalmic Research Center, Labbafinejad Medical Center, Shahid Beheshti University of Medical Sciences, Tehran, Iran https://orcid.org/0000-0002-1459-

6752

Hosein Nouri ( $\square$ Hosein.nouri.2018@gmail.com)

Ophthalmic Research Center, Labbafinejad Medical Center, Shahid Beheshti University of Medical Sciences, Tehran, Iran https://orcid.org/0000-0003-18080443

Jamil Eslamipour

Department of Ophthalmology, Faculty of Medicine, Kurdistan University of Medical Sciences, Sanandaj, Iran

Ebrahim Gerami

Ophthalmic Research Center, Labbafinejad Medical Center, Shahid Beheshti University of Medical Sciences, Tehran, Iran

Hamid Ahmadieh (D Hahmadieh@hotmail.com)

Ophthalmic Research Center, Labbafinejad Medical Center, Shahid Beheshti University of Medical Sciences, Tehran, Iran https://orcid.org/0000-0002-81392661

\section{Research Article}

Keywords: Diabetic retinopathy, Diabetic macular edema, Rho kinase, Fasudil

Posted Date: November 22nd, 2021

DOI: https://doi.org/10.21203/rs.3.rs-1103888/v1

License: @) (7) This work is licensed under a Creative Commons Attribution 4.0 International License. Read Full License 


\section{Abstract}

Purpose: To evaluate the effect of three monthly intravitreal injections of a Rho-associated protein kinase (ROCK) inhibitor (Fasudil, Asahi Kasei Pharma Corporation, Tokyo, Japan) on refractory diabetic macular edema (DME).

Study design: Prospective - clinical

Methods: This interventional case series included 10 eyes of 10 patients with DME unresponsive to at least six previous intravitreal bevacizumab (IVB) injections. Eligible eyes underwent intravitreal injection of $0.025 \mathrm{mg} / 0.05 \mathrm{ml}$ Fasudil. Best-corrected visual acuity (BCVA) and central macular thickness (CMT) were evaluated as functional and anatomical response indicators, respectively.

Results: Mean age was $60.1 \pm 5.1$ (range, 53-68) years. Five cases responded to treatment; two with both anatomical and functional response (reduction of CMT from 521 to 395 and from 390 to 301 microns and improvement of BCVA from 0.3 to 0.1 LogMAR and 0.6 to 0.4 LogMAR, respectively) and three with only functional improvement ( 0.7 to $0.4 ; 0.7$ to 0.4 ; and 0.3 to 0.1 LogMAR). Of note, cases with no significant CMT change had morphologic improvement of retinal microstructure to some extent. No adverse event was observed during the study period.

Conclusion: Monotherapy with intravitreal injection of ROCK inhibitors seems to have moderate visual benefits in eyes with DME refractory to IVB. Such effects may be functionally significant without obvious anatomical improvement.

\section{Introduction}

Diabetic retinopathy is the most prevailing cause of impaired vision and blindness in working-age individuals worldwide; in most cases, diabetic macular edema (DME) is the main reason (1). Optimal therapeutic strategies in managing DME are evolving continuously, and failure of conventional treatments in refractory DME (rDME) underlines the need for new methods. Intravitreal injection of anti-vascular endothelial growth factor (anti-VEGF) agents is the mainstay of DME treatment, though it does not carry thorough visual and anatomical success in a significant proportion of DME cases (2).

Rho/Rho-associated protein kinase (ROCK) signaling pathway inhibitors are seemingly promising in managing several ophthalmic conditions, including DME (3). Our preliminary experience with combined intravitreal injection of a ROCK inhibitor (Fasudil) and bevacizumab in five DME patients who had not responded to previous bevacizumab administrations showed significant visual and structural improvements within six months (4). In another pilot study with a larger sample size, Fasudil administration as adjunctive therapy in rDME brought about significant visual improvement and decreased central macular thickness within four weeks (5). A randomized controlled trial showed that intravitreal injection of Fasudil and bevacizumab could yield more prolonged and more prominent anatomical and visual improvements than bevacizumab alone in center-involved DME (6).

The extent to which monotherapy with ROCK-inhibitors can be of therapeutic value in rDME remains unknown. Therefore, this preliminary interventional case series aimed to investigate the outcome of treatment with Fasudil alone for rDME.

\section{Methods}

In this interventional case series, 10 eyes of 10 patients with rDME were included. General inclusion criteria were, i) best-corrected visual acuity (BCVA) of $20 / 40$ or less, ii) at least six previous intravitreal bevacizumab (IVB) injections with no satisfactory improvement, iii) central macular thickness (CMT) of at least 300 microns. Exclusion criteria were previous intraocular surgery or photocoagulation within the last three months, active proliferative diabetic retinopathy (PDR), uveitis, glaucoma, macular pathologies other than DME, previous vitrectomy, and pregnancy. Patients' demographic information, data on their diabetic retinopathy, associated complications and medications, and history of their previous interventions were obtained.

Three monthly intravitreal injections of Fasudil $0.025 \mathrm{mg} / 0.05 \mathrm{~mL}$ (Asahi Kasei Pharma Corporation, Tokyo, Japan) were planned. Based on individual assessments one month after the initial injection, the decision to continue with two additional monthly injections was made. In cases with excellent response to first or second injection (improvement of vision better than 16/20 or macular thickness of less than 300 microns), further injections were suspended.

Patients were evaluated for intra- or post-operative complications a day and a week after each intravitreal injection. BCVA and CMT, as obtained via optical coherence tomography (Spectralis SD-OCT, Heidelberg Engineering, Heidelberg, Germany), were evaluated at baseline and four weeks after each injection.

The Ethics Committee of the Ophthalmic Research Center permitted the off-label use of Fasudil; patients were informed about the probable risks and benefits associated with this therapeutic approach; signed informed consents were obtained from all participants.

Statistical analyses were performed by SPSS v.26 for windows. Wilcoxon signed-rank test was used to evaluate the statistical significance of changes in BCVA logMAR and CMT values before and after the whole intervention period.

\section{Results}

Patients' mean age and mean diabetes duration (range) were 60.1 (53-68) and 19.9 (16-30) years, respectively. Five eyes had regressed PDR following previous panretinal photocoagulation (PRP), and five had non-proliferative diabetic retinopathy (NPDR). None revealed any sign of inflammation at any given assessment session. Data on patients' diabetic retinopathy stage, current medication(s), number of previous IVB injections, and DME-associated complications are presented in table-1. Mean ( \pm SD) BCVA and CMT at baseline were $0.77( \pm 0.44)$ LogMAR and $482( \pm 177.85)$ microns. No adverse event throughout the study period was noted. Clinical and imaging characteristics of all the cases are summarized in Table- 1 . OCT images of cases $1,2,4,5$, and 6 are presented in Figure-1. 
In the total study population, neither BCVA nor CMT values changes were statistically significant; mean change \pm SD in BCVA was $-0.05 \pm 0.21$ LogMAR $(p=0.49)$ and mean change \pm SD in CMT was $60.6 \pm 146.5$ microns $(p=0.674)$. However, Fasudil administration resulted in a favorable outcome in five eyes:

two with both functional and anatomical improvement (cases No. 1 and 2; see Table-1) and three with functional improvement only (cases No. 4, 7, and 9; see Table-1). As regards case No. 4, in spite of minimal reduction in CMT, morphologic improvement in the foveal contour was noted (see Figure-1). Fasudil administration was discontinued after the first injection in patient No. 4 because BCVA improved favorably to 0.1 LogMAR after the only injection and patient No. 10 due to the patient's refusal to continue the study. No significant improvements were observed in cases No. 3, 5, 6, 8, and 10 (see Table-1).

\section{Discussion}

More than half of DME patients respond well to anti-VEGF therapies; however, inflammation in a significant proportion of cases does not subside with such therapeutic approaches alone (2). Both VEGF-dependent and -independent pathways are suggested to be involved in DME; in rDME, the latter has more pronounced effects than the former (7). VEGF overexpression in rDME triggers downstream pathways involving Rho/ROCK signaling, capable of independently promoting a retinal inflammatory milieu and disrupting the blood-retinal barrier integrity. The delay between VEGF overexpression and Rho/ROCK pathway upregulation may explain the failure of anti-VEGF therapies in controlling rDME (7). Moreover, Rho/ROCK pathway upregulation promotes inflammation and leukocyte adhesion to the endothelial surface (8) and exerts vasoconstrictive and apoptotic effects on endothelial cells via inhibiting endothelial nitric oxide synthesis (9).

We have previously reported the favorable outcome of combined fasudil and bevacizumab intravitreal administration in rDME compared to bevacizumab monotherapy (4), but data on ROCK-inhibitors monotherapy in rDME was lacking. An in-vivo study on Kimba mice has shown that ripasudil (a selective ROCKinhibitor) could suppress the expression of some inflammatory cytokines present in rDME, namely, tumor necrosis factor- $a$ (TNF- $a$ ), MCP-1, and KC (the murine homolog of interleukin-8), whereas bevacizumab suppressed TNF-a and interleukin-6. Ripasudil alone could bring about a significant reduction in retinal thickness; bevacizumab co-administration could synergistically enhance this reduction (7).

The present preliminary study shows a clinically modest effect of fasudil monotherapy in rDME cases resistant to prior standard treatments with bevacizumab; visual improvements with or without anatomical changes were achieved in $50 \%$ of the eyes; however, the changes did not reach statistical significance. In some cases, the functional and anatomical improvements did not necessarily ensue in parallel, i.e., visual improvements were achieved in three eyes with no significant CMT reductions. Such discordances between visual improvements and CMT changes have been described by some previous studies as well (10). For instance, plasma kallikrein inhibition in rDME eyes has shown acceptable visual improvements without significant changes in DME anatomical parameters (11); plasma kallikrein is a serine protease elevated in DME eyes vitreous, capable of increasing vascular permeability and contributing to retinal edema in a VEGF-independent fashion (12). This incongruity may stem from variable edema and ischemia durations with different patterns of influencing CMT and visual acuity; disorganization of the inner retinal layers, in lieu of CMT changes, is suggested to better correspond with visual acuity modifications $(10,13)$.

It is noteworthy that cases without significant CMT change had morphologic improvement of retinal microstructure to some extent (Case No. 4). None of the eyes with baseline BCVA of 20/400 (1.30 LogMAR) or worse $(n=3)$ benefited from Fasudil monotherapy in terms of visual function.

We injected $0.025 \mathrm{mg} / 0.05 \mathrm{~mL}$ fasudil intravitreally and detected no adverse events a day and a week after each injection, in line with previous animal studies (14) and our prior experiences (4-6).

To summarize, intravitreal fasudil monotherapy in rDME eyes unresponsive to previous conventional treatments is safe and has a moderate beneficial effect in improving visual function, not necessarily accompanied by significant anatomical improvement. However, no substantial therapeutic gain may be achieved in patients with severe baseline visual impairments $(B C V A<20 / 400)$.

\section{Declarations}

\section{Conflicts of interest}

Authors declare none.

\section{Funding}

No funding was received for the conduction and documentation of this research.

\section{References}

1. Tan, G. S., Cheung, N., Simó, R., Cheung, G. C., \& Wong, T. Y. (2017). Diabetic macular oedema. The lancet Diabetes \& endocrinology, 5(2), $143-155$.

2. Bressler, S. B., Ayala, A. R., Bressler, N. M., Melia, M., Qin, H., Ferris, F. L., ... Diabetic Retinopathy Clinical Research Network. (2016). Persistent macular thickening after ranibizumab treatment for diabetic macular edema with vision impairment. JAMA ophthalmology, 134(3), $278-285$.

3. Nourinia, R., Nakao, S., Zandi, S., Safi, S., Hafezi-Moghadam, A., \& Ahmadieh, H. (2018). ROCK inhibitors for the treatment of ocular diseases. British Journal of Ophthalmology, 102(1), 1-5.

4. Ahmadieh, H., Nourinia, R., \& Hafezi-Moghadam, A. (2013). Intravitreal fasudil combined with bevacizumab for persistent diabetic macular edema: a novel treatment. JAMA ophthalmology, 131(7), 923-924. 
5. Nourinia, R., Ahmadieh, H., Shahheidari, M. H., Zandi, S., Nakao, S., \& Hafezi-Moghadam, A. (2013). Intravitreal fasudil combined with bevacizumab for treatment of refractory diabetic macular edema; a pilot study. Journal of ophthalmic \& vision research, 8(4), 337.

6. Ahmadieh, H., Nourinia, R., Hafezi-Moghadam, A., Sabbaghi, H., Nakao, S., Zandi, S., ... Akbarian, S. (2019). Intravitreal injection of a Rho-kinase inhibitor (fasudil) combined with bevacizumab versus bevacizumab monotherapy for diabetic macular oedema: a pilot randomised clinical trial. British Journal of Ophthalmology, 103(7), 922-927.

7. Arima, M., Nakao, S., Yamaguchi, M., Feng, H., Fujii, Y., Shibata, K., ... Sonoda, K. H. (2020). Claudin-5 redistribution induced by inflammation leads to antiVEGF-resistant diabetic macular edema. Diabetes, 69(5), 981-999.

8. Lee, H., Lin, C. I., Liao, J. J., Lee, Y. W., Yang, H. Y., Lee, C. Y., ... Wu, H. L. (2004). Lysophospholipids increase ICAM-1 expression in HUVEC through a Gi-and NF-kB-dependent mechanism. American Journal of Physiology-Cell Physiology, 287(6), C1657-C1666.

9. Ming, X. F., Viswambharan, H., Barandier, C., Ruffieux, J., Kaibuchi, K., Rusconi, S., \& Yang, Z. (2002). Rho GTPase/Rho kinase negatively regulates endothelial nitric oxide synthase phosphorylation through the inhibition of protein kinase B/Akt in human endothelial cells. Molecular and cellular biology, 22(24), 8467-8477.

10. Diabetic Retinopathy Clinical Research Network. (2007). Relationship between optical coherence tomography-measured central retinal thickness and visual acuity in diabetic macular edema. Ophthalmology, 114(3), 525-536.

11. Bhatwadekar, A. D., Kansara, V. S., \& Ciulla, T. A. (2020). Investigational plasma kallikrein inhibitors for the treatment of diabetic macular edema: an expert assessment. Expert opinion on investigational drugs, 29(3), 237-244.

12. Kita, T., Clermont, A. C., Murugesan, N., Zhou, Q., Fujisawa, K., Ishibashi, T., ... Feener, E. P. (2015). Plasma kallikrein-kinin system as a VEGF-independent mediator of diabetic macular edema. Diabetes, 64(10), 3588-3599.

13. Gardner, T. W., Antonetti, D. A., Barber, A. J., LaNoue, K. F., Levison, S. W., \& Penn State Retina Research Group. (2002). Diabetic retinopathy: more than meets the eye. Survey of ophthalmology, 47, S253-S262.

14. Kita, T., Hata, Y., Arita, R., Kawahara, S., Miura, M., Nakao, S., ... Ishibashi, T. (2008). Role of TGF- $\beta$ in proliferative vitreoretinal diseases and ROCK as a therapeutic target. Proceedings of the National Academy of Sciences, 105(45), 17504-17509.

\section{Tables}




\begin{tabular}{|c|c|c|c|c|c|c|c|c|c|c|c|c|c|c|}
\hline \multirow{3}{*}{$\begin{array}{l}\text { Case } \\
\text { No. }\end{array}$} & \multirow[t]{3}{*}{ Age/sex } & \multirow{3}{*}{$\begin{array}{l}\begin{array}{l}\text { DM } \\
\text { duration/ }\end{array} \\
\text { Current } \\
\text { Tx }\end{array}$} & \multirow{3}{*}{$\begin{array}{l}\text { Index } \\
\text { eye/ } \\
\text { DR } \\
\text { stage/ } \\
\text { rDME }\end{array}$} & \multirow{3}{*}{$\begin{array}{l}\text { No. of } \\
\text { previous } \\
\text { IVB }\end{array}$} & \multicolumn{6}{|c|}{ Snellen BCVA (logMAR) } & \multicolumn{4}{|l|}{ CMT $(\mu \mathrm{m})$} \\
\hline & & & & & Baseline & 1 & 2 & 3 & Last & $\begin{array}{l}\text { Change } \\
(\%)\end{array}$ & Baseline & 1 & 2 & 3 \\
\hline & & & & & & & & & & & & & & \\
\hline \multirow[t]{2}{*}{1} & \multirow[t]{2}{*}{$68 / M$} & \multirow{2}{*}{$\begin{array}{l}20 / \text { Ins + } \\
\text { met }\end{array}$} & \multirow{2}{*}{$\begin{array}{l}\text { OD/ } \\
\text { Mod. } \\
\text { NPDR/- }\end{array}$} & \multirow[t]{2}{*}{$>10$} & $20 / 80$ & \multirow{2}{*}{$\begin{array}{l}20 / 100 \\
(0.70)\end{array}$} & \multirow{2}{*}{$\begin{array}{l}20 / 160 \\
(0.90)\end{array}$} & \multirow{2}{*}{$\begin{array}{l}20 / 50 \\
(0.40)\end{array}$} & \multirow{2}{*}{$\begin{array}{l}20 / 50 \\
(0.40)\end{array}$} & \multirow{2}{*}{$\begin{array}{l}-0.20 \\
(-33.4 \%)\end{array}$} & \multirow[t]{2}{*}{390} & \multirow[t]{2}{*}{306} & \multirow[t]{2}{*}{300} & \multirow[t]{2}{*}{301} \\
\hline & & & & & $(0.60)$ & & & & & & & & & \\
\hline \multirow[t]{2}{*}{2} & \multirow[t]{2}{*}{$61 / F$} & \multirow{2}{*}{$\begin{array}{l}16 / \operatorname{lns}+ \\
\text { met }\end{array}$} & \multirow{2}{*}{$\begin{array}{l}\text { OD/ } \\
\text { Mod. } \\
\text { NPDR/- }\end{array}$} & \multirow[t]{2}{*}{5} & $20 / 40$ & $20 / 30$ & $20 / 40$ & $20 / 50$ & $20 / 25$ & -0.20 & \multirow[t]{2}{*}{521} & \multirow[t]{2}{*}{455} & \multirow[t]{2}{*}{432} & \multirow[t]{2}{*}{395} \\
\hline & & & & & $(0.30)$ & $(0.18)$ & $(0.30)$ & $(0.40)$ & $(0.10)$ & $(-66.7 \%)$ & & & & \\
\hline 3 & $64 / M$ & 22/Met & OS/Sev. & 5 & $20 / 400$ & $20 / 630$ & $20 / 630$ & $20 / 630$ & $20 / 630$ & 0.2 & 439 & 528 & 528 & 647 \\
\hline & & & NPDR/ & & $(1.30)$ & $(1.50)$ & $(1.50)$ & $(1.50)$ & $(1.50)$ & $(15.4 \%)$ & & & & \\
\hline & & & rDME & & & & & & & & & & & \\
\hline 4 & $57 / F$ & 21/Met & OS/ & 5 & $20 / 40$ & $20 / 25$ & - & - & $20 / 25$ & -0.20 & 309 & 307 & - & - \\
\hline & & & NPDR/- & & $(0.30)$ & $(0.10)$ & & & $(0.10)$ & $(-66.7 \%)$ & & & & \\
\hline 5 & $62 / M$ & 17/Met & & $>10$ & $20 / 40$ & $20 / 40$ & $20 / 40$ & $20 / 70$ & $20 / 70$ & 0.24 & 408 & 468 & 743 & 692 \\
\hline & & & $\begin{array}{l}\text { Sev. } \\
\text { NPDR/- }\end{array}$ & & $(0.30)$ & & & $(0.54)$ & $(0.54)$ & $(80.0 \%)$ & & & & \\
\hline 6 & $58 / F$ & 16/Met & OS/ & 10 & $20 / 400$ & $20 / 630$ & $20 / 630$ & $20 / 630$ & $20 / 630$ & 0.2 & 769 & 744 & 652 & 744 \\
\hline & & & $\begin{array}{l}\text { reg. } \\
\text { PDR/ } \\
\text { rDME }\end{array}$ & & $(1.30)$ & $(1.50)$ & $(1.50)$ & $(1.50)$ & $(1.50)$ & $(15.4 \%)$ & & & & \\
\hline 7 & $53 / F$ & $20 /$ Ins & OS/ & 5 & $20 / 100$ & $20 / 40$ & $20 / 40$ & $20 / 40$ & $20 / 50$ & -0.30 & 367 & 362 & 362 & 341 \\
\hline & & & $\begin{array}{l}\text { PDR/ } \\
\text { rDME }\end{array}$ & & $(0.70)$ & & & $(0.30)$ & $(0.40)$ & $(-42.9 \%)$ & & & & \\
\hline 8 & $56 / \mathrm{M}$ & $19 /$ Ins & OS/ & 10 & $20 / 630$ & $20 / 630$ & $20 / 630$ & $20 / 630$ & $20 / 630$ & 0.0 & 827 & 936 & 727 & 988 \\
\hline & & & $\begin{array}{l}\text { PDR/ } \\
\text { rDME }\end{array}$ & & $(1.50)$ & $(1.50)$ & $(1.50)$ & $(1.50)$ & $(1.50)$ & $(0.0 \%)$ & & & & \\
\hline 9 & $55 / M$ & 18/Met & OD/ & 8 & $20 / 100$ & $20 / 50$ & $20 / 25$ & $20 / 50$ & $20 / 50$ & -0.30 & 457 & 444 & 423 & 444 \\
\hline & & & $\begin{array}{l}\text { reg. } \\
\text { PDR }\end{array}$ & & $(0.70)$ & & $(0.10)$ & & & $(-42.9 \%)$ & & & & \\
\hline 10 & $67 / M$ & 30/Met & OS/ & $>10$ & $20 / 100$ & $20 / 100$ & - & - & $20 / 100$ & 0.0 & 333 & 567 & - & - \\
\hline & & & $\begin{array}{l}\text { PDR/ } \\
\text { rDME }\end{array}$ & & $(0.70)$ & & & & & $(0.0 \%)$ & & & & \\
\hline
\end{tabular}

Table1. Patients' characteristics, history of Treatments, and comparison of best-corrected visual acuity (BCVA) and central macular thickness (CMT) values at baseline, after each session of Fasudil administration, and at the last follow-up.

Abbreviations: BCVA, best-corrected visual acuity; CMT, central macular thickness; DM, diabetes mellitus; DR, diabetic retinopathy; F, female; Ins, insulin; IVB, intravitreal bevacizumab; logMAR, the logarithm of the minimum angle of resolution; M, male; Met, metformin; Mod., moderate; NPDR, non-proliferative diabetic retinopathy; OD, oculus dexter; OS, oculus sinister; rDME, refractory diabetic macular edema; Reg., regressed; Sev., severe; Tx, treatment

\section{Figures}




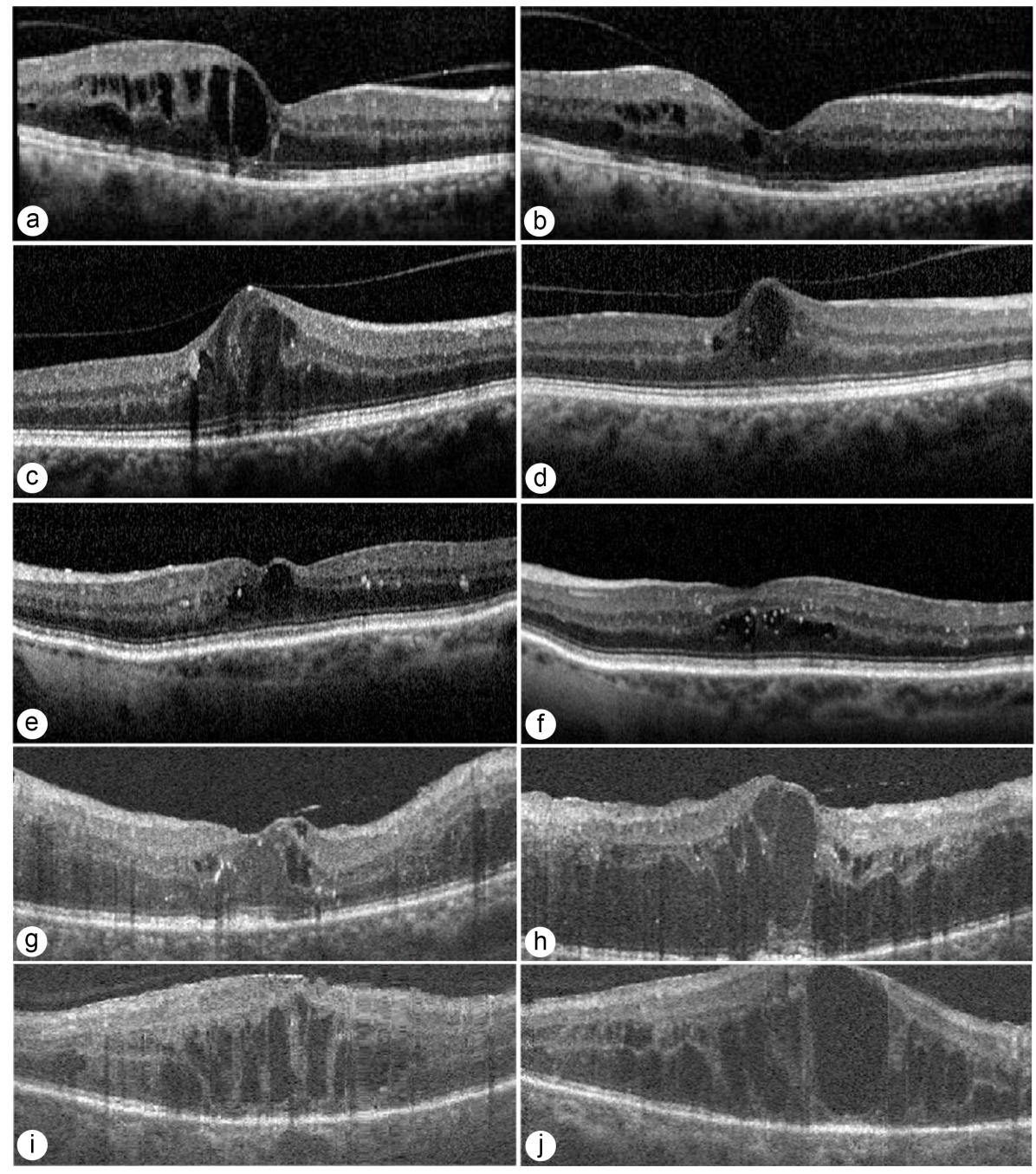

Figure 1

Baseline (a, c, e, g, i) and post-treatment (b, d, f, h, j) structural OCT images of five cases (No. 1, 2, 4, 5, 6), respectively. 